\title{
A guideline for the prevention and treatment of thromboembolism in COVID-19 patients
}

\author{
Saeed Safari ${ }^{1,2}$, Mahmoud Yousefifard $^{3 *}$ \\ 'Proteomics Research Center, Shahid Beheshti University of Medical Sciences, Tehran, Iran \\ ${ }^{2}$ Emergency Department, Shohadye Tajrish Hospital, Shahid Beheshti University of Medical Sciences, Tehran, Iran \\ ${ }^{3}$ Physiology Research Center, Iran University of Medical Sciences, Tehran, Iran
}

\section{Dear Editor}

The coronavirus disease 2019 (COVID-19) was first emerged from Wuhan, China, in late 2019, and has since been spreading progressively all around the world. Its prevalence is climbing increasingly and almost all countries worldwide are confronting this pandemic. As of April 11,2020, reports obtained about the management of COVID-19 patients indicate that the mortality rate of the disease is around $5 \%$ with consideration of the active cases and $21 \%$ of the closed cases (1).

Preliminary experiences had indicated that the leading cause of death in COVID-19 patients was severe hypoxia following acute respiratory distress syndrome $(2,3)$; however, the gradually increasing information regarding the pathophysiology of the disease has shown that angiotensin-converting enzyme 2 (ACE2) is a cellular receptor for the severe acute respiratory syndromecoronavirus 2 (SARS-CoV-2) (4). The distribution of ACE2 receptor is widespread in the human body and is found in abundance in various tissue cells, including epithelial cells, vascular endothelial cells, kidney cells, brain tissue cells and etc. (5). Therefore, it is hypothesized that ARDS may not be the only cause of death in patients. Furthermore, recent findings demonstrating the inconsistency between the clinical manifestations of some patients with their imaging and laboratory findings has reinforced the mentioned hypothesis $(3,6)$. For instance, Zhou et al reported that $18 \%$ of non-severe cases and 3\% of severe cases of COVID-19 had normal presentations in their chest radiography and computed tomography (CT) scans (7).
The existing literature regarding COVID-19 indicates that coagulopathy is a common disorder among these patients and is significantly related to the mortality rate and severity of the disease. The presence of ACE2 in blood vessels causes the vascular endothelial cells to be a convenient host for SARS-CoV-2. As a result, recent studies have reported numerous vascular thromboses occurring in various tissues such as lungs, brain and heart of COVID-19 patients. For example, a study on 184 proved COVID-19 cases admitted to ICU indicated a 31\% prevalence of vascular thrombosis among the patients. Between different kinds of thrombosis, pulmonary embolism was the most prevalent (81\%) (8). Furthermore, a cohort study conducted on 183 patients demonstrated a relation between coagulopathy and poor prognosis among COVID-19 patients (9). Also, a case control study reported that anticoagulant treatment can reduce 28 -day mortality rate in patients with sepsis induced coagulopathy (10).

Such research findings indicate that coagulopathies are of utmost importance in COVID-19 patients. Hence, the present study aims to provide an anticoagulant treatment protocol which can be used in COVID-19 patients suspected or at risk of having thrombosis.

The first step in the mentioned protocol is identifying the COVID-19 patients at risk of thromboembolism. Therefore, in this step, risk factors for thromboembolism in the patients should be monitored. These risk factors include inherited coagulation disorders, immobilization, pregnancy, contraceptive consuming, obesity and overweight, smoking, cardiovascular disorders, inflammatory bowel disease, family history of thrombosis,

Received: 9 May 2020; Accepted: 29 August 2020; Published online: 13 September 2020

*Corresponding author: Mahmoud Yousefifard; Physiology Research Center, School of Medicine, Iran University of Medical Sciences, Hemmat highway, Tehran, Iran; Tel: +982186704771, Email: yousefifard.m@iums.ac.ir;

Competing interests: None reported.

Funding information: There is none to be declared.

Citation: Safari S, Yousefifard M. A guideline for the prevention and treatment of thromboembolism in COVID-19 patients. Journal of Emergency Practice and Trauma 2021, 7(1): 4-6. doi: 10.34172/jept.2020.30. 
recent history of thrombosis or pulmonary embolism, old age, cancer, recent trauma, recent surgery and etc. Presence of the mentioned risk factors can cause a COVID-19 patient to be at risk of coagulopathy.

The second step is to identify symptoms indicating of thrombosis. Symptoms which demonstrate an underlying thrombosis are divided into four main groups: (a) The primary and most important symptoms of thrombosis in COVID-19 patients are related to pulmonary embolism, since based on the reports, $81 \%$ of the thromboses are occurred in the lungs of COVID-19 patients (8). Symptoms related to pulmonary embolism include dyspnea which cannot be justified by the extent of pulmonary involvement as observed in CT scan, decreased arterial oxygen saturation levels down to less than $85 \%$, decreased oxygen saturation levels despite treatment and improvement of findings in chest CT scans, haemoptysis, pleuritic chest pain and the presence of findings in electrocardiography (ECG) indicating pulmonary embolism (S1Q3T3). (b) Symptoms related to stroke should also be taken into consideration. These symptoms include decreased level of consciousness which cannot be justified by the level of oxygen saturation and general hypoxemia, delirium, sudden speech impediment, decreased motor ability, impaired vision, inability to walk, confusion, imbalance, and sudden severe headache. (c) Symptoms related to cardiac ischemia are also of great importance. These symptoms include severe chest pain, changes in the EEG raising doubt over cardiac ischemia, increased levels of cardiac enzymes such as Troponin I. (d) Finally, symptoms related to deep thrombosis, such as pain in the limbs, redness, warmth of the limbs to the touch, worsening of the leg pain with bending of the foot, muscle crumps in the limbs and screen discoloration.

The third step includes monitoring of d-dimer levels. The d-dimer test should be a routine evaluation in COVID-19 patients. There is no consensus on the optimum cut-off for d-dimer in COVID-19 patients. Studies on COVID-19 have proposed a wide range cut offs for d-dimer (from $1500 \mathrm{ng} / \mathrm{mL}$ to $5300 \mathrm{ng} / \mathrm{mL})(11-13)$. In designing the present protocol, based on expert opinion a d-dimer of $1000 \mathrm{ng} / \mathrm{mL}$ was used. This cut-off can be modified based on the local clinical experience in other countries. If d-dimer levels are less than $1000 \mathrm{ng} / \mathrm{mL}$, low molecular weight heparin (LMWH) should be given as a prophylaxis at a daily dose of $40 \mathrm{mg}$. Also, if d-dimer levels are above

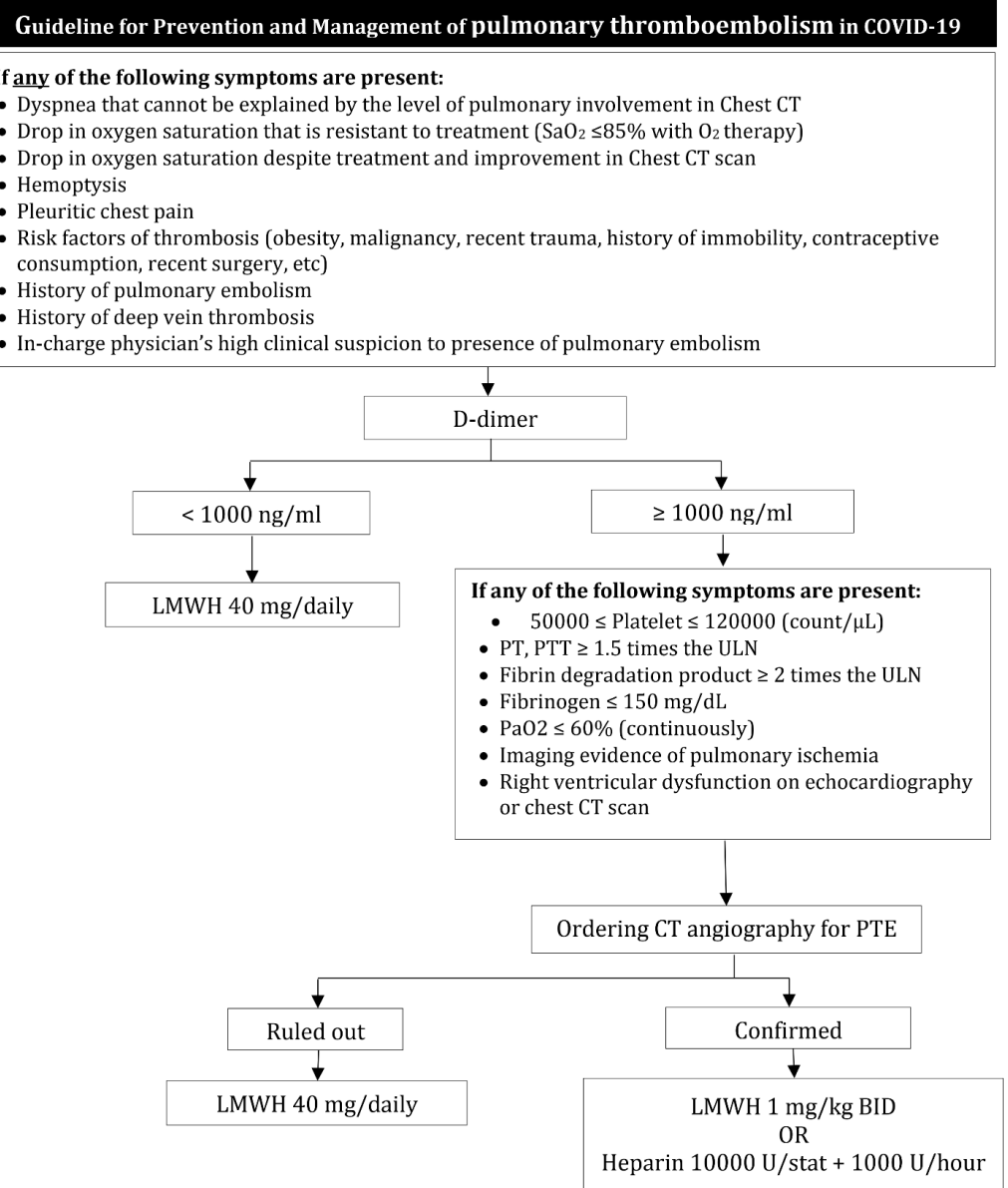

Figure 1. Recommended flowchart for the prevention and treatment of COVID-19 suspected thromboembolism patients. CT: Chest computed tomography scan without contrast; LMWH: Low molecular weight heparin; PTE: pulmonary thromboembolism; ULN: Upper limit of normal. 
$1000 \mathrm{ng} / \mathrm{mL}$, further evaluations are recommended. In cases of any coagulation disorders observed in the blood test, $\mathrm{PaO} 2 \leq 60 \%$, imaging evidences of thromboembolism, right ventricular dysfunction in echocardiography or ECG changes, and CT angiography of the lungs are recommended, and if the pulmonary artery thrombosis is confirmed, LMWH $80 \mathrm{mg}$ /daily should be prescribed for the patients. Clearly, in cases of suspected thrombotic related disorders in brain and heart, diagnostic procedures such as MRI and coronary angiography are recommended. If vascular thromboses are ruled out, prophylaxis treatment (LMWH $40 \mathrm{mg}$ /daily) is recommended for the patients.

As we said previously, $81 \%$ of thrombotic events in COVID-19 patients are related to pulmonary embolism. Therefore, the proposed protocol is a recommendation for the management of patients suspected or at risk of having pulmonary thromboembolism as the most frequent thrombotic event in COVID-19 cases (Figure 1).

Authors' contributions

SS and MY had equal contributions.

Ethical issues

Not applicable.

\section{Acknowledgment}

All the efforts and suggestions of Dr. Nikooghadam and Dr. Mehdi Pishgahi in improving the scientific quality of the guideline are appreciated.

\section{References}

1. World Metrics. COVID-19 coronavirus outbreak. [cited 2020 Mar 17]; Available from: https://www.worldometers. info/coronavirus/.

2. Guan WJ, Ni ZY, Hu Y, Liang WH, Ou CQ, He JX, et al. Clinical characteristics of coronavirus disease 2019 in China. N Engl J Med 2020; 382(18): 1708-20. doi: 10.1056/ NEJMoa2002032.

3. Wang Y, Yao L, Zhang J, Tang P, Ye Z, Shen X, et al. Clinical characteristics and laboratory indicator analysis of 69 COVID-19 pneumonia patients in Suzhou, China. Research Square; 2020. doi: 10.21203/rs.3.rs-20968/v1.

4. Guo J, Huang Z, Lin L, Lv J. Coronavirus disease 2019
(COVID-19) and cardiovascular disease: a viewpoint on the potential influence of angiotensin囚 converting enzyme inhibitors/angiotensin receptor blockers on onset and severity of severe acute respiratory syndrome coronavirus 2 infection. J Am Heart Assoc 2020; 9(7): e016219. doi: 10.1161/jaha.120.016219.

5. Hamming I, Timens W, Bulthuis ML, Lely AT, Navis G, van Goor H. Tissue distribution of ACE2 protein, the functional receptor for SARS coronavirus. A first step in understanding SARS pathogenesis. J Pathol 2004; 203(2): 631-7. doi: 10.1002/path.1570.

6. Bernheim A, Mei X, Huang M, Yang Y, Fayad ZA, Zhang $\mathrm{N}$, et al. Chest $\mathrm{CT}$ findings in coronavirus disease-19 (COVID-19): relationship to duration of infection. Radiology 2020; 295(3): 200463. doi: 10.1148/ radiol.2020200463.

7. Zhou F, Yu T, Du R, Fan G, Liu Y, Liu Z, et al. Clinical course and risk factors for mortality of adult inpatients with COVID-19 in Wuhan, China: a retrospective cohort study. Lancet 2020; 395(10229): 1054-62. doi: 10.1016/s01406736(20)30566-3.

8. KlokFA, Kruip M, van der Meer NJM, Arbous MS, Gommers $\mathrm{D}$, Kant KM, et al. Incidence of thrombotic complications in critically ill ICU patients with COVID-19. Thromb Res 2020; 191: 145-7. doi: 10.1016/j.thromres.2020.04.013.

9. Tang N, Li D, Wang X, Sun Z. Abnormal coagulation parameters are associated with poor prognosis in patients with novel coronavirus pneumonia. J Thromb Haemost 2020; 18(4): 844-7. doi: 10.1111/jth.14768.

10. Tang N, Bai H, Chen X, Gong J, Li D, Sun Z. Anticoagulant treatment is associated with decreased mortality in severe coronavirus disease 2019 patients with coagulopathy. J Thromb Haemost 2020; 18(5): 1094-9. doi: 10.1111/ jth.14817.

11. Yao Y, Cao J, Wang Q, Liu K, Luo Z, Yu K, et al. D-dimer as a biomarker for disease severity and mortality in COVID-19 patients: a case control study. Research Square; 2020. doi: 10.21203/rs.3.rs-20850/v1.

12. Zhou Y, Yang Z, Guo Y, Geng S, Gao S, Ye S, et al. A new predictor of disease severity in patients with COVID-19 in Wuhan, China. medRxiv; 2020. doi: 10.1101/2020.03.24.20042119.

13. Cui S, Chen S, Li X, Liu S, Wang F. Prevalence of venous thromboembolism in patients with severe novel coronavirus pneumonia. J Thromb Haemost 2020; 18(6): 1421-4. doi: 10.1111/jth.14830. 